\title{
Nonintegrability of the Classical Zeeman Hamiltonian
}

\author{
M. Kummer, ${ }^{1 \star}$ A.W. Saenz ${ }^{2 \star \star}$ \\ ${ }^{1}$ Department of Mathematics, University of Toledo, Toledo, Ohio 43606, USA \\ ${ }^{2}$ Department of Physics, Catholic University, Washington, D.C. 20064, USA and Naval Research \\ Laboratory, Washington, D.C. 20375, USA
}

Received: 24 February 1993/in revised form: 20 September 1993

\begin{abstract}
We prove that the Hamiltonian $H$ of the three dimensional hydrogen atom in a uniform static magnetic field $\mathbf{B}$ does not have an integral which (i) is real analytic on the phase space $\mathscr{M}$ of the system; (ii) is in involution with the component $M_{3}$ of the angular momentum along $\mathbf{B}$; (iii) is functionally independent of $H$ and $M_{3}$ and (iv) has a meromorphic (single-valued) extension to the complexification of $\mathscr{M}$ in $\mathbb{C}^{6}$. This follows from the fact that the Hamiltonian $K_{M}$ of two degrees of freedom obtained by fixing $M_{3}$ at certain nonzero values $M$ and reducing $H$ w.r. to the rotational symmetry about the magnetic field, has a complexification which is nonintegrable in the Ziglin sense. We prove this nonintegrability by demonstrating that for each such $M$ the monodromy group of the normal variational equation along a certain complexified phase curve of $K_{M}$ is not Ziglin, using Churchill and Rod's adaptation of Kovacic's algorithm to the Ziglin analysis. Analogous arguments prove that the Hamiltonian of the Størmer problem is nonintegrable in the same sense.
\end{abstract}

\section{Introduction}

There is intense interest in the classical and quantum mechanical behavior of the hydrogen atom in a static magnetic field (the "magnetized hydrogen atom" for short), and the physics literature on this subject is enormous (see e.g., [1-5] and the bibliographical information contained therein). The topic is important both from a fundamental physical viewpoint and because of its atomic physics and astrophysics applications. From a fundamental point of view, a large part of interest in the classical magnetized hydrogen atom arises because of the belief among many physicists (supported by numerous numerical and formal analytical arguments) that chaos in classical systems entails chaos, in some sense, in the corresponding

* Supported by Contract \# N00173-90-9706 from the Naval Research Laboratory and (consecutively) a summer faculty fellowship from the University of Toledo.

$\star \star$ Supported by a fellowship from the Max Planck Institute in Stuttgart. 
quantized systems, as discussed by, e.g., Percival $[6,7]$ and Berry [8-10], and in [11] and [12] (see [11] and [12] for bibliography).

There is strong numerical evidence for the occurrence of chaos in the classical magnetized hydrogen atom and its quantum counterpart in strong fields $[1-5,13]$. This is prima facie evidence of the nonintegrability of this classical system. On the other hand numerical studies [1-3] suggest the existence of an "approximate" third integral of this system, i.e., independent of the energy and angular momentum integrals. However, no rigorous mathematical study settling the question of integrability of this classical system has been published heretofore.

As a first step toward understanding rigorously the underlying mechanism for chaotic behavior of the three-dimensional magnetized hydrogen atom, we will prove that the classical Hamiltonian $H$ of this system is nonintegrable, in the sense that there does not exist a real analytic integral $F$ of $H$ in involution with the angular momentum component $M_{3}$ in the magnetic field direction, which is functionally independent of $H$ and $M_{3}$ and which has a global meromorphic (one-valued) extension to the complexified phase space to which $H$ can be analytically continued as a single valued function. We will prove this by showing that for some real nonzero value $M$ of $M_{3}$, the Hamiltonian $K_{M}$ of two degrees of freedom, obtained by reduction of the Hamiltonian $H$ of this system with respect to the rotational symmetry about the direction of the magnetic field has a complexification which is nonintegrable in the Ziglin sense defined below.

Using the methods of this paper, we have also proved an analogous rigorous nonintegrability result for the Hamiltonian system describing the motion of an electron in a magnetic dipole field (the Størmer problem). This result will be reported in more detail in a future publication. Numerical studies [14-16] have suggested, but not proved, the nonexistence of global analytic third integrals for this system.

Returning to the problem of the classical magnetized hydrogen atom, its Hamiltonian is defined by

$$
H(X, Y)=\frac{\mathbf{Y}^{2}}{2}-\frac{1}{r}+\gamma M_{3}+\frac{1}{2} \gamma^{2}\left(X_{1}^{2}+X_{2}^{2}\right),
$$

on the phase space

$$
\mathscr{M}:=\left\{(\mathbf{X}, \mathbf{Y}) \in \mathbb{R}^{3} \times \mathbb{R}^{3}:\left(X_{1}^{2}+X_{2}^{2}\right)>0\right\},
$$

where $\mathbf{X}=\left(X_{1}, X_{2}, X_{3}\right), \mathbf{Y}=\left(Y_{1}, Y_{2}, Y_{3}\right)$. This is the Hamiltonian of the quadratic Zeeman effect in atomic units with $M_{3}=X_{1} Y_{2}-X_{2} Y_{1}$ the component of the angular momentum along the magnetic field, assumed to be parallel to the $X_{3}$-axis, $r=\sqrt{X_{1}^{2}+X_{2}^{2}+X_{3}^{2}}=\|\mathbf{X}\|$ the distance of the electron from the nucleus, and $\gamma:=\frac{B \mu_{0} a_{0}}{e^{2}}$. Here $B$ is the magnitude of the magnetic field, and $\mu_{0}, a_{0}, e$ are Bohr magneton, the Bohr radius, and the nuclear charge, respectively. In what follows, $\mathbf{B}$, and hence $\gamma$ will be assumed to be nonzero.

We give $\mathscr{M}$ the structure of a real symplectic six-dimensional manifold by equipping it with the non-degenerate two-form $d \Theta_{0}$, where $\Theta_{0}$ is the one-form

$$
\Theta_{0}:=\mathbf{Y} \cdot d \mathbf{X}
$$

Since $H$ is invariant under rotations about the $X_{3}$-axis, $M_{3}$ is in involution with $H$ on $\mathscr{M}$, and the Hamiltonian $H$ can be reduced immediately to one with two 
degrees of freedom. This is best done by introducing the canonical extension of cylindrical coordinates, in the phase space $\mathscr{M}$,

$$
X:=X_{1}+i X_{2}=\rho e^{i \phi}, \quad Y:=Y_{1}+i Y_{2}=\left[\pi+i \frac{M}{\rho}\right] e^{i \phi},
$$

where, $M=M_{3}$. The canonical one-form in these coordinates becomes

$$
\Theta_{0}:=\operatorname{Re}(\bar{Y} d X)+Y_{3} d X_{3}=\pi d \rho+M d \phi+Y_{3} d X_{3} .
$$

We denote by $K_{M}$ the Hamiltonian (1.1) in the new variables with the trivial paramagnetic term $\gamma M$ subtracted (which simply amounts to rescaling the energy). Thus $K_{M}: U \rightarrow \mathbb{R}$ is given by

$$
K_{M}\left(\rho, \pi, X_{3}, Y_{3}\right)=\frac{1}{2}\left(\pi^{2}+\frac{M^{2}}{\rho^{2}}+Y_{3}^{2}\right)-\frac{1}{\sqrt{\rho^{2}+X_{3}^{2}}}+\frac{\gamma^{2}}{2} \rho^{2} .
$$

The domain

$$
U:=(0, \infty) \times \mathbb{R}^{3}
$$

of variation of the new variables $\left(\rho, \pi, X_{3}, Y_{3}\right)$ is viewed as symplectic fourmanifold equipped with the two-form $d \Theta$, where

$$
\Theta:=\pi d \rho+Y_{3} d X_{3} \text {. }
$$

An extensive mathematical literature is devoted to the question of integrability of Hamiltonians of two degrees of freedom and, as we shall see, contains results directly relevant to the nonintegrability of our Hamiltonian (1.1). The pioneering work on the nonintegrability of analytic Hamiltonians was carried out by Ziglin [17]. An analytic Hamiltonian $K$ defined on a complex four-dimensional symplectic manifold $X$ will be said to be integrable in the sense of Ziglin if there exists a function $F$ which is (i) meromorphic on $X$; (ii) in involution with $K$; (iii) functionally independent of $K$ in an $X$-neighborhood of a complexified nonequilibrium phase curve of the vector field associated with $K$, this curve having been maximally extended in complex time. Henceforth integrability of a Hamiltonian of two degrees of freedom will always be understood in this sense, in contrast with that of our original Hamiltonian $H$ (with three degrees of freedom) which will always have the meaning mentioned previously.

The crucial idea of Ziglin's approach is to study the influence of integrability of $K$ on the monodromy group associated with the (reduced) normal variational equation (NVE) along some complexified nonequilibrium solution $\Gamma$, the latter being viewed as a Riemann surface. Analytic continuation of solutions of the NVE along cycles on $\Gamma$ based on some point $z \in \Gamma$ defines a representation of the homotopy group of $\Gamma$ as a subgroup of the general linear group $G L(2, \mathbb{C})$ which acts on the fiber of the (reduced) normal bundle above $z$. The image of this representation is the monodromy group $G$ of the NVE. For a Hamiltonian $K$ of two degrees of freedom $G$ is always a subgroup of $S L(2, \mathbb{C})$. If $K$ is integrable $G$ leaves invariant a rational function of the coordinates along the fiber in question. Subgroups of $S L(2, \mathbb{C})$ having this property have been called Ziglin by subsequent authors, who have extended and refined Ziglin's work. In this connection we refer to papers of Ito [18], Yoshida [19-21], and Rod, Churchill, and Baider [22-26].

For all real nonzero values of $M$ our Hamiltonian $K_{M}$ has an analytic single valued extension $\widetilde{K}_{M}$ to a complex four-dimensional manifold $\widetilde{U}$ (defined in Sect. II) 
and the nonintegrability of $H$ will be proved by showing that $\tilde{K}_{M}$ is not integrable for $M$ varying in a certain open interval. This will be done by using Churchill and Rod's tailoring [22] of Kovacic's algorithm [27] to the Ziglin analysis. This algorithm was originally devised to solve symbolically on a computer second order linear homogeneous ODEs whose coefficients are rational functions. The algorithm is implemented on the computer program MAPLE. It is a procedure that allows the computer to search systematically for a Liouvillian solution, i.e., a solution built up using exponentials, integrals, and functions algebraic over the field $\mathbb{C}(t)$ of rational functions of the independent variable $t$. It turns out that if the differential equation has a nonzero Liouvillian solution, then it also has a solution of type $\exp \int \theta$, with $\theta$ algebraic over the field $\mathbb{C}(t)$. Depending on the precise nature of $\theta$ the algorithm distinguishes three cases that also differ by the nature of the so-called differential Galois group, associated with the differential equation. Consider the field $\mathscr{F}$, obtained by extending the field $\mathbb{C}(t)$ by solutions of the linear differential equation and their derivatives of all orders. Of course, if the differential equation has order $n$, extending the field $\mathbb{C}(t)$ by $n$ linearly independent solutions and their first $n-1$ derivatives already generates the field $\mathscr{F}$. The differential Galois group is the group of all those automorphisms of $\mathscr{F}$ that leave $\mathbb{C}(t)$ pointwise fixed (see e.g. $[28,29]$ ). Since there is a close connection between the monodromy group and the differential Galois group of a second order ODE of Fuchsian type, i.e., having only regular singularities, (see [30]), Kovacic's algorithm can be used to determine whether the monodromy group of such an equation is not Ziglin. Combining it with Ziglin's analysis [17], it becomes a powerful tool for deciding integrability questions in Hamiltonian systems of two degrees of freedom. This idea has been recently presented and worked out in detail by Churchill and Rod in [22]. We have applied the approach in [22] to the NVE along the complexification $\tilde{\Gamma}_{E, M} \subset \tilde{U}$ of a phase curve of $\tilde{K}_{M}$, induced by a certain periodic solution $\Gamma_{E, M}$ of the associated planar problem obtained by setting $X_{3}=Y_{3}=0$. Notice that on $\Gamma_{E, M}$ energy $E$ and angular momentum $M_{3}$ are fixed at the values $E$ and $M$, respectively. The monodromy group of this NVE is then embedded into that of an ODE of Fuchsian type, to which Kovacic's algorithm is applied, with the result that the Galois group of its normal form is the whole group $S L(2, \mathbb{C})$. This immediately implies that the monodromy group of this Fuchsian equation is not Ziglin. Using this fact together with a symmetry argument, we show that the monodromy group of the original NVE is not Ziglin, thus proving our main theorem by virtue of the corollary to Lemma 2.1. This theorem asserts that $H$ is not integrable, and more precisely that there does not exist a function with properties (i)-(iv) stated therein.

It should be stressed that this is the first physically interesting example in which the method proposed by Churchill and Rod [22] is successfully applied. Unlike the examples in the literature designed to illustrate this method, the present application is highly non-trivial, since one is forced to run through the entire machinery of Kovacic's algorithm and prove that none of the three cases in which a Liouvillian solution exists can be realized.

The organization of this paper is as follows. In Sect. II, we state our main theorem together with two key auxiliary results, namely, Lemma 2.1 and its corollary alluded to above. The fact that the monodromy group of the Fuchsian ODE into which we embed the above NVE is not Ziglin is shown in Sect. III. That the monodromy group of the NVE itself is not Ziglin is proved in Sect. IV. Appendix A contains the statements and proofs of Lemmas A1-A3, which are 
needed to prove Lemma 2.1. To make the paper as self-contained as possible, we have summarized Churchill's and Rod's version of Kovacic's algorithm in Appendix B.

\section{Preliminary Considerations, Statement of Main Theorem, and Reduction of its Proof to that of Proving that the Monodromy group of a Suitable NVE is not Ziglin}

The present section consists of three subsections. Subsection A describes a family of phase curves $\Gamma_{E, M}$ of $K_{M}$, complexified versions $\tilde{\Gamma}_{E, M}$ of which play an essential role in this paper. Our main theorem is stated in Subsect. B. In Subsect. C, we state and prove Lemma 2.1 and its corollary, which is essential in the proof of that theorem.

$A$. In order to construct the family of phase curves $\Gamma_{E, M}$ we notice that the submanifold $X_{3}=0, Y_{3}=0$ is invariant under the flow induced by the Hamiltonians $H, K_{M}$ in their respective phase spaces $\mathscr{M}$ and $U$. Restricting $K_{M}$ to this submanifold and fixing its value to $E \in \mathbb{R}$, we obtain the $\mathrm{ODE}$

$$
\frac{1}{2} \pi^{2}=\frac{1}{2} \dot{\rho}^{2}=E-V_{M}(\rho)
$$

where

$$
V_{M}(\rho):=\frac{1}{2} \frac{M^{2}}{\rho^{2}}-\frac{1}{\rho}+\frac{1}{2} \gamma^{2} \rho^{2} .
$$

In the case when $M \neq 0, V_{M}(\rho)$ has an absolute minimum at the point $\rho_{M}$, where $\rho_{M}$ is the unique positive solution of the equation

$$
M^{2}=\rho+\gamma^{2} \rho^{4} \Leftrightarrow \frac{M^{2}}{\rho^{2}}=\frac{1}{\rho}+\gamma^{2} \rho^{2} .
$$

In this case, the stable equilibrium point ( $\rho=\rho_{M}, \pi=0, X_{3}=0, Y_{3}=0$ ) with energy $E_{\min }^{+}(M):=\min _{\rho>0} V_{M}(\rho)$ is "surrounded" by the family of planar periodic solutions

$$
\Gamma_{E, M}=\left\{\left(\rho(t), \pi(t), X_{3}(t)=0, Y_{3}(t)=0\right) \subset U, t \in \mathbb{R}\right\},
$$

which persist for all values of the energy $E>E_{\min }^{+}(M)$. Here, $\rho(t), \pi(t)=\dot{\rho}(t)$ are solutions of (2.1) whose $E, M$-dependence has been suppressed. For $M=0, V_{M}(\rho)$ has no equilibrium points on $(0, \infty)$. The integral curves $\Gamma_{E, 0}$ of $K_{M}$ for $M=0$ and energy $E \in \mathbb{R}$ corresponds to motions in which the electron "falls" into the nucleus, i.e.,

$$
\Gamma_{E, 0}=\left\{\left(\rho(t), \pi(t), X_{3}(t)=0, Y_{3}(t)=0\right) \subset U,|t| \leqq T_{E}\right\},
$$

where $\rho(t), \pi(t)$ (with their $E$-dependence suppressed) satisfy (2.1) with $M=0$. In (2.5), $T_{E}$ is the (finite) time required by an electron of energy $E$ and angular momentum zero starting from rest to "fall' into the nucleus.

In what follows we fix $E$ at a value bigger than $E_{\min }^{+}(M)$ and keep $M$ different from zero. We write Eq. (2.1) in the form

$$
\pi^{2} \rho^{2}+G(\rho)=0
$$


where $G(\rho)$ is the following fourth degree polynomial in $\rho$ :

$$
G(\rho):=2 \rho^{2}[-E+V(\rho)]=\gamma^{2} \rho^{4}-2 E \rho^{2}-2 \rho+M^{2} .
$$

Generically, the polynomial $G(\rho)$ has four distinct roots in the complex plane, exactly two of which are real positive. More precisely, this condition is satisfied iff

$$
E \in J_{M} \subset \mathbb{R},
$$

where

$$
J_{M}=\left(E_{\min }^{+}(M), \infty\right) \backslash\left\{E_{\min }^{-}(M)\right\}
$$

with $E_{\min }^{-}(M):=\min _{\rho<0} V_{M}(\rho)>E_{\min }^{+}(M)$. If we write

$$
G(\rho)=\gamma^{2}\left(\rho-a_{1}\right)\left(\rho-a_{2}\right)\left(\rho-a_{3}\right)\left(\rho-a_{4}\right),
$$

where $a_{1}, a_{2}\left(a_{1}>a_{2}>0\right)$ are the two positive real roots then $a_{3}, a_{4}$ are conjugate complex for $E_{\min }^{+}(M)<E<E_{\min }^{-}(M)$ and real negative for $E>E_{\min }^{-}(M)$. In any case, for these values of $E$ all four roots are mutually distinct.

From the point of view of algebraic geometry (2.6) can be viewed as an algebraic curve $\hat{\Gamma}_{E, M}$ in $\mathbb{C}^{2}$, which upon setting $p=\pi \rho$ takes on the elliptic form:

$$
p^{2}+G(\rho)=0 \text {. }
$$

Indeed, by inverting the function $s(\rho):=\int_{a_{2}}^{\rho} \frac{d \xi}{\sqrt{-G(\xi)}}$, defined on the Riemann surface $\mathbb{T}^{1}$ corresponding to $\sqrt{-G(\rho)}$ which, as is well known, is topologically a two-torus, we obtain an elliptic function $\rho: \mathbb{C} / \mathscr{L} \rightarrow \mathbb{T}^{1}$. Together with its derivative, it yields a parametrization of $\hat{\Gamma}_{E, M}$ :

$$
\rho=\rho(s), \quad p=\rho^{\prime}(s), \quad \text { i.e., } \pi(s)=\frac{\rho^{\prime}(s)}{\rho(s)} .
$$

Here, $\mathscr{L}$ is the period lattice (with one period real). Notice that $\rho(s)$ is an even function in $s$ and accordingly $\rho^{\prime}(s)$ is odd, so that the curve becomes manifestly invariant under time inversion: $s \rightarrow-s$. However, $s$ is not the physical time $t$, but the so-called Levi-Civita time (or the eccentric anomaly of the ellipses which our periodic solutions asymptotically for small values of the magnetic field represent). It is related to physical time by means of the formula:

$$
\frac{d t}{d s}=\rho(s) \Rightarrow t(s)=\int_{0}^{s} \rho(\sigma) d \sigma .
$$

Thus, $t(s)$ is an odd function of $s$, so that $s \rightarrow-s$ implies $t \rightarrow-t$.

$B$. The principal result of this paper is as follows.

Main Theorem. The Hamiltonian $H$ in (1.1) is not integrable, in the sense that there does not exist a function $F: \mathscr{M} \rightarrow \mathbb{R}$ with the following properties:

(i) $F$ is real analytic.

(ii) $F$ is in involution with $H, M_{3}$ on $\mathscr{M}$.

(iii) $d F, d H, d M_{3}$ are linearly independent at some point $p_{0}:=\left(\mathbf{X}_{0}, \mathbf{Y}_{0}\right) \in \mathscr{M}$.

(iv) $F$ has a meromorphic extension $\hat{F}$ to $\hat{\mathscr{M}}$. 
Here, $\hat{\mathscr{M}}$ is the complexification of $\mathscr{M}$, i.e.,

$$
\hat{\mathscr{M}}:=\left\{(\mathbf{X}, \mathbf{Y}) \in \mathbb{C}^{6}: X_{1}^{2}+X_{2}^{2}+X_{3}^{2} \neq 0, X_{1}^{2}+X_{2}^{2} \neq 0\right\}
$$

Remarks.

(1) Plainly, this theorem excludes the existence of polynomial integrals of $H$ in involution with $M_{3}$ and functionally independent of $H, M_{3}$.

(2) It does not exclude the existence of real analytic integrals of $H$ which have multiple-valued extensions to some open subset of $\mathbb{C}^{6}$.

(3) Since linear independence is an open relation we can (and will) assume that the point $p_{0}$ whose existence is postulated in (iii) of the main theorem belongs to $\mathscr{M}_{M_{0}}$ with $M_{0} \neq 0$.

Here,

$$
\mathscr{M}_{M}:=\left\{(\mathbf{X}, \mathbf{Y}) \in \mathscr{M}: X_{1} Y_{2}-X_{2} Y_{1}=M\right\} .
$$

C. In order to state Lemma 2.1 and its corollary, whose significance for this paper was mentioned informally in Sect. $I$ it is necessary to define a maximal singlevalued analytic extension of $K_{M}$, as well as to give other pertinent definitions. The complication occurring in the definition of this extension is caused by the presence of the square root $\sqrt{\rho^{2}+X_{3}^{2}}$ in the definition of $K_{M}$ (see (1.6)), which does not allow us to extend this Hamiltonian maximally in a single-valued way to open subsets $\mathbb{C}^{4}$. Consider the complexification

$$
\hat{U}:=\left\{\left(\rho, \pi, X_{3}, Y_{3}\right) \in \mathbb{C}^{4}: \rho \neq 0, \rho^{2}+X_{3}^{2} \neq 0\right\}
$$

of the domain $U \subset \mathbb{R}^{4}$ in (1.7). Let $\tilde{U}$ be the double cover of $\hat{U}$ equipped with the relative topology of $\mathbb{C}^{5}$ and realized as the part of the zero set of the polynomial $\rho^{2}+X_{3}^{2}-w^{2}$ in $\mathbb{C}^{5}$ which lies over $\hat{U}$ (coordinates $\left.\left(\rho, \pi, X_{3}, Y_{3}, w\right)\right)$. The corresponding covering map pr: $\tilde{U} \rightarrow \hat{U}$ is defined by $\operatorname{pr}\left(\rho, \pi, X_{3}, Y_{3}, w\right)=\left(\rho, \pi, X_{3}, Y_{3}\right)$ and $\tilde{U}$ is equipped with the pullback via pr of a complexification of the two-form $d \Theta$ in (1.8). For each $M \in \mathbb{R}$ we define the extension $\widetilde{K}_{M}$ of the Hamiltonian $K_{M}$ to $\tilde{U}$ by

$$
\tilde{K}_{M}\left(\rho, \pi, X_{3}, Y_{3}, w\right):=\frac{1}{2}\left(\pi^{2}+\frac{M^{2}}{\rho^{2}}+Y_{3}^{2}\right)-\frac{1}{w}+\frac{1}{2} \gamma^{2} \rho^{2} .
$$

Hence $\tilde{K}_{M}$ is holomorphic and single valued on $\tilde{U}$. In the remainder of this section We assume $M \in \mathbb{R} \backslash\{0\}, E \in J_{M}$. We also introduce the complexified phase curve of $\widetilde{K}_{M}$ as an embedding of the algebraic curve $\widehat{\Gamma}_{E, M}$ into $\tilde{U}$ :

$$
\tilde{\Gamma}_{E, M}:=\left\{(\rho, \pi, 0,0, \rho) \subset \tilde{U}:(\rho, \pi) \in \hat{\Gamma}_{E, M}\right\} .
$$

We now prove

Lemma 2.1. Assume that a function $F$ exists having properties (i)-(iv) stated in the main Theorem. Then if $M$ belongs to a certain open interval $\mathscr{J}$ the Hamiltonian $\widetilde{K}_{M}$ is integrable in the Ziglin sense. More precisely, for these values of $M$ there exists a function $\widetilde{F}_{M}$ which is meromorphic in $\tilde{U}$, in involution with $\widetilde{K}_{M}$, and functionally independent of $\tilde{K}_{M}$.

Proof. It is based on Lemmas A1-A3 of Appendix A. By assumptions (i) and (ii) of the main theorem, and Lemma A1 (a), there exists a real analytic function 
$F_{M}: U \rightarrow \mathbb{R}$ independent of $\phi$ and defined by $F_{M}\left(\rho, \pi, X_{3}, Y_{3}\right)=F(\mathbf{X}, \mathbf{Y})\left((\mathbf{X}, \mathbf{Y}) \in \mathscr{M}_{M}\right)$. Moreover, by (i), (ii), (iv) of the main theorem, and Lemma A2, for all nonzero values of $M$ there exists a meromorphic function $\widetilde{F}_{M}$, defined by $\widetilde{F}_{M}=\operatorname{pr}^{*} \widehat{F}_{M}$ in terms of the meromorphic extension $\hat{F}_{M}$ of $F_{M}$ to $\hat{U}$ proved in Lemma A2.

By (i), (ii) of the main Theorem, Lemmas A1 (b), and Lemma A3, $\widetilde{F}_{M}$ on $\tilde{U}$ is in involution with $\tilde{K}_{M}$. By assumption (iii) of the main theorem and Remark (3) following it, $d F, d H, d M_{3}$ are linearly independent at a point $p_{0}:=\left(\mathbf{X}_{0}, \mathbf{Y}_{0}\right) \in \mathscr{M}_{M_{0}}$ with $M_{0} \neq 0$. Hence, by Lemma A1 (c), we see that $d F_{M}$ and $d K_{M}$ are linearly independent at the corresponding point in $U \subset \hat{U}$ provided $M$ is restricted to the interval $\mathscr{J}$. It immediately follows from this observation that the pair of functions $F_{M}, K_{M}$ and therefore also the pair $\tilde{F}_{M}, \tilde{K}_{M}$ for these values of $M$ is functionally independent and the proof of the present lemma is complete.

Lemma 2.1 is of interest here because of the following

Corollary. In order for the main theorem to hold, if suffices that the monodromy group of the NVE along $\widetilde{\Gamma}_{E, M}$ for some real nonzero $M$ be not of Ziglin type, i.e., not leave invariant a rational function of the fiber coordinates.

Proof. Follows from Lemma 2.1 and a result of Ziglin (see Proposition on p. 183 in the first Ref. [17]).

\section{Principal Argument of the Proof of the Main Theorem}

In this section, we fix $M \neq 0, E \in J_{M}$. We will first derive the NVE along the solution $\tilde{\Gamma}_{E, M}$, then embed this NVE into a Fuchsian ODE, and finally show that the monodromy group of the latter equation is not Ziglin.

Expanding the right side of (2.16) in the variables $X_{3}, Y_{3}$ about a point $(\rho, \pi, 0,0, \rho) \in \tilde{U}$ under the assumption that $\left|X_{3}\right|<|\rho|$, we obtain:

$$
\begin{aligned}
\tilde{K}_{M}\left(\rho, \pi, X_{3}, Y_{3}, w\right. & \left.=\rho+X_{3}^{2} / 2 \rho+O\left(\frac{X_{3}^{4}}{\rho^{3}}\right)\right) \\
& =\frac{1}{2} \pi^{2}+V_{M}(\rho)+\frac{1}{2}\left(\frac{1}{\rho^{3}} X_{3}^{2}+Y_{3}^{2}\right)+O\left(\frac{X_{3}^{4}}{\rho^{5}}\right) .
\end{aligned}
$$

It follows from (3.1) that in terms of complexified physical time $t$ the NVE along $\tilde{\Gamma}_{E, M}$ is governed by the time dependent Hamiltonian $h=\frac{1}{2}\left(\eta^{2}+\frac{1}{\rho^{3}} \xi^{2}\right)$, where $(\xi, \eta):=\left(X_{3}, Y_{3}\right)$ are (canonically conjugate) fiber coordinates of the reduced normal bundle and $\rho$ is viewed a function of complex time via ODE (2.1):

$$
\ddot{\xi}+\frac{1}{\rho^{3}} \xi=0 \text {. }
$$

Rather than studying the monodromy group of (3.2) directly, we embed it into that of a Fuchsian equation. This strategy was first used by Yoshida in [21] in the special case of the hypergeometric ODE and later generalized by Baider, Churchill, and Rod [23]. In the present context this will be accomplished by a switch of the 
independent variable from $t$ to $\rho$. Using primes to denote derivatives with respect to $\rho$, Eq. (3.2) is transformed into

$$
\xi^{\prime \prime}+\frac{\ddot{\rho}}{\dot{\rho}^{2}} \xi^{\prime}+\frac{1}{\rho^{3} \dot{\rho}^{2}} \xi=0 .
$$

Since $\dot{\rho}^{2}+\rho^{-2} G(\rho)=0 \Rightarrow 2 \ddot{\rho} \dot{\rho}+\left[\rho^{-2} G^{\prime}(\rho)-2 \rho^{-3} G(\rho)\right] \dot{\rho}=0 \Rightarrow \ddot{\rho}+\left[\frac{1}{2} \rho^{-2} G^{\prime}(\rho)\right.$ $\left.-\rho^{-3} G(\rho)\right]=0$, we can write

$$
\xi^{\prime \prime}+p(\rho) \xi^{\prime}+q(\rho) \xi=0
$$

with

$$
p(\rho):=\frac{1}{2} \frac{G^{\prime}(\rho)}{G(\rho)}-\frac{1}{\rho}, \quad q(\rho):=-\frac{1}{G(\rho) \rho} .
$$

In accordance with an earlier remark (see (2.10)) for the present values of angular momentum and energy, the four roots of the polynomial

$$
G(\rho)=\gamma^{2}\left(\rho-a_{1}\right)\left(\rho-a_{2}\right)\left(\rho-a_{3}\right)\left(\rho-a_{4}\right),
$$

are non-zero and pairwise distinct, and two of them, say $a_{1}, a_{2}$ are positive. The pairwise distinctness of $a_{1}, a_{2}, a_{3}, a_{4}$ is a necessary and sufficient condition for the ODE (3.4) to be Fuchsian. Using (3.5), (3.6), and the pairwise distinctness of the numbers $a_{0}:=0, a_{1}, a_{2}, a_{3}, a_{4}$, it follows that the partial fraction decomposition (B2) in Appendix B assumes the form

$$
p(\rho):=\sum_{k=0}^{4} \frac{A_{k}}{\rho-a_{k}}, \quad q(\rho)=-\frac{1}{G(\rho) \rho}=\sum_{k=0}^{4} \frac{C_{k}}{\rho-a_{k}},
$$

where $\sum_{k=0}^{4} C_{k}=\frac{1}{2 \pi i} \oint_{C_{\infty}} q(z) d z=0$ is satisfied since $\lim _{\rho \rightarrow \infty} \frac{1}{G(\rho)}=0\left(C_{\infty}=\right.$ circle of infinite radius). Moreover,

$$
A_{0}=-1, A_{1}=A_{2}=A_{3}=A_{4}=\frac{1}{2}
$$

and

$$
B_{k}=0 \quad(k=0,1,2,3,4)
$$

by (B2) and (3.7), and by (B6) in view of (3.5),

$$
A_{\infty}:=\sum_{k=0}^{4} A_{k}=1, B_{\infty}:=\frac{1}{2 \pi i} \oint_{C_{\infty}} q(z) z d z=0, \text { since } \lim _{\rho \rightarrow \infty} \frac{\rho}{G(\rho)}=0 .
$$

Henceforth in this section, the subscript $k$ (resp., $\sigma$ ) stands for any integer from 0 to 4 (resp., 1 to 4 ). By (B5), (B6), and (3.8) we have in the present case:

$$
\begin{gathered}
\beta_{0}=\frac{3}{4}, \quad \beta_{\sigma}=-\frac{3}{16}, \quad \beta_{\infty}=-\frac{1}{4} ; \\
\alpha_{0}^{ \pm}=\frac{3}{2},-\frac{1}{2} ; \quad \alpha_{\sigma}^{ \pm}=\frac{3}{4}, \frac{1}{4} ; \quad \alpha_{\infty}^{ \pm}=\frac{1}{2} .
\end{gathered}
$$


We are now ready to apply Kovacic's algorithm as presented in Appendix B to study the monodromy group $G_{E, M}$ of the Fuchsian Equation (3.4). This algorithm determines whether any of the mutually exclusive cases I, II, or III defined in Appendix B is realized. If none of them is realized, then $G_{E, M}$ is not Ziglin.

Case I. By (3.9), the only way the degree $d_{I}$ of the monic polynomial $P_{I}$ in rule (3) of Case I of the Appendix can be a nonnegative integer is to choose minus signs $s(k)$ in (B8). For this choice, $d_{I}=0$ by (B8) and (3.9), i.e. $P_{I} \equiv 1$. Defining $\omega(\rho)$ by (B10), we see by (3.7)-(3.9) that $\omega=\frac{1}{2} p(\rho)$ in the present situation. Thus, by (B9), the only way Case I can be realized in this situation is for $\omega^{\prime}(\rho)+\omega^{2}(\rho)-r(\rho) \equiv 0$. Since $r(\rho)=-q(\rho)+\frac{1}{4} p(\rho)^{2}+\frac{1}{2} p^{\prime}(\rho)$ (see (B4)), we find that $\omega^{\prime}(\rho)+\omega^{2}(\rho)-r(\rho)=q(\rho)$, i.e., an expression which is obviously not identically zero because $\lim _{\rho \rightarrow 0} \rho$ $q(\rho)=-1 / M^{2}<0$ (by (2.7) and (3.5)). Hence we have ruled out Case I.

Case II. By (3.9), the sets (B11) are $E_{0}=\{-2,2,6\}, E_{\sigma}=\{1,2,3\}, E_{\infty}=\{2\}$ in the present case. Since $e_{\infty}=2$ the only way integers $e_{0}, e_{\sigma}$ can be chosen from $E_{0}$ and $E_{\sigma}$, so that the right side of $(\mathrm{B} 12)$ is a non-negative integer is to choose $e_{0}=-2$ and $e_{\sigma}=1$. With this selection, the degree $d_{I I}$ of the monic polynomial $P_{I I}$ in assertion (3) of Case II is zero, i.e., $P_{I I} \equiv 1$. This choice is legitimate according to rule (2) of case II since not all the chosen integers are even. Recalling (3.7) and (3.8a) it leads to $\theta=p=2 \omega$ for $\theta$ in (B14). Thus, according to (B13), Case II can be realized in the present situation only if the identity

$$
\begin{aligned}
0 & \equiv \theta^{\prime \prime}+3 \theta^{\prime} \theta+\theta^{3}-4 r \theta-2 r^{\prime} \equiv\left(2 \theta+\frac{d}{d \rho}\right)\left(\theta^{\prime}+\frac{1}{2} \theta^{2}-2 r\right) \\
& =2\left(2 \theta+\frac{d}{d \rho}\right)\left(\omega^{\prime}+\omega^{2}-r\right)
\end{aligned}
$$

holds, i.e., only if $\left(2 p(\rho)+\frac{d}{d \rho}\right) q(\rho) \equiv 0$. Since $q(\rho)=-\frac{1}{\rho M^{2}}+\tilde{q}(\rho)$, where $\tilde{q}(\rho)$ is analytic at $\rho=0$, we easily find that the coefficient of the term proportional to $\frac{1}{\rho^{2}}$ in the expression $\left(2 p(\rho)+\frac{d}{d \rho}\right) q(\rho)$ becomes $\frac{3}{M^{2}}$. The fact that this is not zero rules out case II.

Case III. In what follows we fix $n=4,6,12$. By (3.9), the sets (B15) are

$$
\begin{aligned}
& F_{0}(n):=\left\{6+\frac{24 e}{n}: e=0, \pm 1, \pm 2, \ldots, \pm \frac{n}{2}\right), \\
& F_{\sigma}(n):=\left\{6+\frac{6 e}{n}: e=0, \pm 1, \pm 2, \ldots, \pm \frac{n}{2}\right), \\
& F_{\infty}(n):=\{6\}
\end{aligned}
$$

in the case under discussion. Therefore we are forced to choose $f_{\infty}(n)=6$. Choosing the smallest integers $f_{0}(n), f_{\sigma}(n)$ from $F_{0}(n)$ and $F_{\sigma}(n)$, namely $f_{0}(n)=-6, f_{\sigma}(n)=3$ yields the biggest integer for the degree $d_{I I I}(n)$ of the monic polynomial $P_{I I I}(n)$ in rule (3) of case III. Since this is zero we find $P_{I I I}(n) \equiv 1$. 
Specialized to the present case and with the $f_{k}(n)$ 's chosen as above, definitions (B17) (B19) yield:

$$
\theta_{n}=\frac{n}{2} p(\rho), \quad S=\frac{1}{\gamma^{2}} \rho G(\rho)
$$

where we also used (3.7) and the fact that $a_{0}=0$ and that the $a_{\sigma}$ 's are poles of $r(\rho)$. By (3.5) and (3.11),

$$
\begin{aligned}
S(\rho) \theta_{n}(\rho) & =\frac{n}{2} p(\rho) \rho \frac{1}{\gamma^{2}} G(\rho)=\frac{n}{2 \gamma^{2}}\left[\frac{1}{2} G^{\prime}(\rho) \rho-G(\rho)\right], \\
r(\rho) & =-q(\rho)+\frac{1}{4} p(\rho)^{2}+\frac{1}{2} p^{\prime}(\rho) \\
& =\frac{1}{G(\rho) \rho}+\frac{1}{4}\left(\frac{1}{2} \frac{G^{\prime}(\rho)}{G(\rho)}-\frac{1}{\rho}\right)^{2}+\frac{1}{4} \frac{G(\rho) G^{\prime \prime}(\rho)-G^{\prime}(\rho)^{2}}{G(\rho)^{2}}+\frac{1}{2 \rho^{2}} \\
S^{2}(\rho) r(\rho) & =\frac{1}{\gamma^{4}}\left[-k(\rho) G(\rho)-\frac{3}{16} G^{\prime}(\rho)^{2} \rho^{2}+\frac{1}{4} G(\rho) G^{\prime \prime}(\rho) \rho^{2}\right]
\end{aligned}
$$

where

$$
k(\rho):=-\rho-\frac{3}{4} G(\rho)+\frac{1}{4} G^{\prime}(\rho) \rho=\frac{\gamma^{2}}{4} \rho^{4}+\frac{E}{2} \rho^{2}-\frac{3}{4} M^{2} .
$$

Using (3.12), (3.13) and the fact that now $P_{I I I}(n) \equiv 1$, the polynomials $P_{i}$ in (B18) $(i=n, n-1, n-2, \ldots, 0,-1)$ are seen to be defined recursively as follows:

$$
\begin{aligned}
P_{n} \equiv & -1, \\
P_{i-1}= & -\frac{1}{\gamma^{2}} G(\rho) \rho P_{i}^{\prime}+\frac{1}{\gamma^{2}}\left[(n-i)\left(G(\rho)+\rho G^{\prime}(\rho)\right)+\frac{n}{2}\left(G(\rho)-\frac{1}{2} \rho G^{\prime}(\rho)\right)\right] P_{i} \\
& +\frac{(n-i)(i+1)}{\gamma^{4}}\left[K(\rho) G(\rho)+\frac{3}{16} G^{\prime}(\rho)^{2} \rho^{2}-\frac{1}{4} G^{\prime \prime}(\rho) G(\rho) \rho^{2}\right] P_{i+1}
\end{aligned}
$$

or

$$
\begin{aligned}
P_{i-1}= & -\frac{1}{\gamma^{2}} G(\rho) \rho P_{i}^{\prime}+\frac{1}{\gamma^{2}}\left[\left(\frac{3}{2} n-i\right) G(\rho)+\left(\frac{3}{4} n-i\right) \rho G^{\prime}(\rho)\right] P_{i} \\
& +\frac{(n-i)(i+1)}{\gamma^{4}}\left[k(\rho) G(\rho)+\frac{3}{16} G^{\prime}(\rho)^{2} \rho^{2}-\frac{1}{4} G^{\prime \prime}(\rho) G(\rho) \rho^{2}\right] P_{i+1}
\end{aligned}
$$

Write

$$
P_{i}(0)=-c_{i} a^{n-i}, \quad P_{i}^{\prime}(0)=-d_{i} b a^{n-i}(i=n, n-1, n-2, \ldots,-1),
$$

with

$$
a:=\frac{1}{\gamma^{2}} G(0)=\frac{M^{2}}{\gamma^{2}}, \quad b:=\frac{G^{\prime}(0)}{\gamma^{2} a}=-\frac{2}{\gamma^{2} a},
$$


so that

$$
k(0)=-\frac{3}{4} a \gamma^{2}
$$

and

$$
c_{n}=1, \quad c_{n+1}=0, d_{n}=d_{n+1}=0 .
$$

Setting $\rho=0$ in (3.14b) and using (3.15)-(3.17), we obtain recursively:

$$
c_{i-1}=\left(\frac{3}{2} n-i\right) c_{i}-\frac{3}{4}(n-i)(i+1) c_{i+1}, \quad(i=n, n-1, n-2, \ldots, 1,0) .
$$

Moreover, comparing terms linear in $\rho$ on both sides of the recursion relations (3.14b) and again using (3.15) and (3.16) we find:

$$
\begin{aligned}
d_{i-1} a^{n-i+1} b= & -a^{n-i+1} b d_{i}+a\left[\left(\frac{3}{2} n-i\right)+\left(\frac{3}{4} n-i\right)\right] b c_{i} a^{n-i} \\
& +\left(\frac{3}{2} n-i\right) a^{n-i+1} b d_{i} \\
& -(n-i)(i+1) \frac{3}{4}\left[a^{2} d_{i+1} a^{n-i-1} b+c_{i+1} b a^{2} a^{n-i-1}\right],
\end{aligned}
$$

or

$$
\begin{aligned}
d_{i-1} & =\left(\frac{3}{2} n-i-1\right) d_{i}+\left(\frac{9}{2} n-2 i\right) c_{i}-\frac{3}{4}(n-i)(i+1)\left[c_{i+1}+d_{i+1}\right] \\
& =\left(\frac{3}{2} n-i-1\right) d_{i}+c_{i-1}+\left(\frac{3}{4} n-i\right) c_{i}-\frac{3}{4}(n-i)(i+1) d_{i+1} .
\end{aligned}
$$

With the aid of a programmable calculator, we found from (3.18) $c_{-1}=0$, but $d_{-1} \geqq 210$, for $n=4,6,12$, respectively. This result together with (3.15) and the fact that $a$ and $b$ are nonzero, entails that $P_{-1} \not \equiv 0$ in all three cases. Hence, we have ruled out Case III.

\section{Conclusion of the Proof of the Main Theorem}

In the last section we proved that for $M \neq 0, E \in J_{M}$ the monodromy group of the Fuchsian equation (3.4) is not Ziglin. In this section we employ a symmetry argument to conclude that the same is true for the NVE (3.2). According to the corollary to Lemma 2.1, this suffices to draw the conclusion of the main theorem.

Since $\operatorname{pr}_{1}: \hat{\Gamma}_{E, M} \rightarrow \mathbb{C}$ defined by $\operatorname{pr}_{1}(\rho, p)=\rho$ branches of order two over $\rho=a_{1}, a_{2}, a_{3}, a_{4}$ for $M \neq 0$ it is clear that lifts of basic loops about these points are not loops on $\hat{\Gamma}_{E, M}$. Only loops that encircle these points twice, lift to basic loops on the curve. In view of the definition (2.17) this implies that the monodromy group of our NVE along $\tilde{\Gamma}_{E, M}$ is isomorphic only to a subgroup of index two of the monodromy group of our Fuchsian equation. However, since our NVE is invariant under the group $\mathbb{Z}_{2}$ defined by time reversal, it can be interpreted as a differential equation on the curve $\tilde{\Gamma}_{E, M} / \mathbb{Z}_{2}$, and with this interpretation its monodromy group 
becomes isomorphic to the one of the Fuchsian equation. According to Ziglin's main theorem any meromorphic integral of the vector field associated with $K_{M}$ gives rise to a nonconstant rational function $\Phi$ in the fiber-coordinates $\xi, \eta$ which is invariant under the monodromy group of our NVE. However, since the Hamiltonian of our NVE is an even function in $\eta$ it is no restriction to assume that the rational invariant has the same property. Indeed, decomposing $\Phi$ into even and odd part, both parts are invariants separately. If the even part should be constant the square of the odd part is a nonconstant rational invariant which is even in $\eta$. However, such a function is an invariant of the monodromy group of our NVE viewed as living on $\tilde{\Gamma}_{E, M} / \mathbb{Z}_{2}$.

The same conclusion also follows as a special case from Corollary 1.3 of Theorem 1.2 of [22] or Corollary 4.26 of [31]. Therefore, the property of the monodromy group of our Fuchsian equation not being Ziglin suffices to draw the conclusion of the theorem.

Acknowledgment. The authors would like to thank D.L. Rod for suggesting that they use Kovacic's algorithm to decide the integrability as well as for useful discussions. In addition the authors gratefully acknowledge the help of R. Churchill and A. Baider for clarifying some finer points of their understanding of Ziglin's notion of integrability, and M. Singer for having clarified for them the precise nature of a Liouvillian solution. Of course, any errors that still remain are the authors' responsibility. Last but not least, the second author wishes to thank T. Regelmann for having checked the correctness the numerical computations which enabled us to rule out Case III of Kovacic's algorithm (see end of Sect. III).

\section{Appendix A: Auxiliary Results for Proving Lemma 2.1}

In this appendix, we state and prove Lemmas A1-A3, which are used in the proof of Lemma 2.1 in Sect. II. In what follows $\{A, B\}$ will denote the Poisson brackets corresponding to the symplectic structure on which the functions $A$ and $B$ are defined. Henceforth we fix $M_{3}=M \neq 0$ and note that according to the transformation (1.4) the point $\left(\rho, \pi, X_{3}, Y_{3}\right)$ of $U$ is in one-one correspondence with a circle on the hypersurface $\mathscr{M}_{M}:=\left\{(\mathbf{X}, \mathbf{Y}) \in \mathscr{M}: X_{1} Y_{2}-X_{2} Y_{1}=M\right\}$ of $\mathscr{M}$. In other words: $\mathscr{M}_{M} \rightarrow U$ is a circle bundle with total space $\mathscr{M}_{M}$ and base $U$.

Lemma A1. (a) The formula

$$
F(\mathbf{X}, \mathbf{Y})=F_{M}\left(\rho, \pi, X_{3}, Y_{3}\right)\left((\mathbf{X}, \mathbf{Y}) \in \mathscr{M}_{M}\right) .
$$

determines a real analytic function $F_{M}: U \rightarrow \mathbb{R}$.

(b) $\left\{F_{M}, K_{M}\right\}\left(\rho, \pi, X_{3}, Y_{3}\right)=\{F, H\}(\mathbf{X}, \mathbf{Y})=0\left((\mathbf{X}, \mathbf{Y}) \in \mathscr{M}_{M}\right)$.

(c) If $d F, d H$ and $d M_{3}$ are linearly independent at some point $\left(\mathbf{X}_{0}, \mathbf{Y}_{0}\right) \in \mathscr{M}_{M_{0}}$ then $d F_{M}$, and $d K_{M}$ are linearly independent at the corresponding point in $U$ for all $M$ in an open interval $\mathscr{J}$ about $M_{0}$.

Proof. The facts (a) and (b) are immediate consequences of the expression (1.5) of the symplectic structure on $\mathscr{M}$ in the canonically extended cylindrical coordinates (see transformation (1.4)). (c) follows from the computation:

$$
\begin{aligned}
d F & =d_{U} F_{M}+\frac{\partial F}{\partial M} d M, d H=d_{U} K_{M}+\frac{\partial K}{\partial M} \partial M \Rightarrow d F \wedge d H \wedge d M \\
& =d_{U} F_{M} \wedge d_{U} K_{M} \wedge d M
\end{aligned}
$$


where $d_{U}$ is the exterior derivative involving only the coordinates of $U$. Since linear independence is an open relation our statement (c) follows.

Lemma A2. If $F$ is a real analytic function on $\mathscr{M}$ with meromorphic extension $\hat{F}$ to $\hat{\mathscr{M}}$ and is independent of $\phi$ then the function $F_{M}$ obtained from $F$ by fixing $M_{3}$ at a real value $M$ is real analytic on $U$ with meromorphic extension $\hat{F}_{M}$ to $\hat{U}$.

Proof. We will assume that $F$ and therefore $\hat{F}$ is not identically zero, for otherwise there is nothing to prove. Since $F$ is independent of $\phi, F(\mathbf{X}, \mathbf{Y})=F\left(\rho, 0, X_{3}, \pi\right.$, $\left.M / \rho, Y_{3}\right)((\mathbf{X}, \mathbf{Y}) \in \mathscr{M})\left(\right.$ set $\phi=0$ in (1.4)). We define $\hat{F}_{0}$ by $\hat{F}_{0}(x, M)=\hat{F}\left(\rho, 0, X_{3}, \pi\right.$, $\left.M / \rho, Y_{3}\right)$ at all $(x, M) \in \hat{U} \times \mathbb{C}$ at which the rhs is defined, with $x=\left(\rho, \pi, X_{3}, Y_{3}\right)$. Therefore $\hat{F}_{0}$ is meromorphic and not identically zero on $\hat{U} \times \mathbb{C}$ since $\hat{F}$ has these properties on $\hat{\mathscr{M}}$. Hence $\hat{U} \times \mathbb{C}$ can be covered by a system $\mathscr{A}$ of open balls such that on each member $\alpha$ of $\mathscr{A}$ the function $\hat{F}_{0}$ has a representation

$$
\hat{F}_{0}\left(x, M_{3}\right)=\frac{f_{\alpha}\left(x, M_{3}\right)}{g_{\alpha}\left(x, M_{3}\right)},
$$

where $f_{\alpha}, g_{\alpha}$ are analytic on $\alpha$, and relatively prime, so that $g_{\alpha}\left(x, M_{3}\right)$ does not vanish identically on $\alpha$. For each real $M$, we define an extension $\hat{F}_{M}(x):=\hat{F}_{0}(x, M)$ of $F_{M}$. We now fix $M \in \mathbb{R}$ and assume that $F_{M} \neq 0$ since otherwise we are done. To prove that $\hat{F}_{M}$ is meromorphic on $\hat{U}$, it suffices to show that $g_{\alpha}(x, M) \neq 0$ on each non-empty ball $\alpha_{M}:=\{x:(x, M) \in \alpha\} \subset \hat{U}(\alpha \in \mathscr{A})$. This inequality is obvious if $\alpha_{M}$ intersects the real domain $U$, because of the assumed real analyticity of $F$. We show $g_{\alpha}(x, M) \neq 0$ in general, even if $\alpha_{M}$ does not contain any real points. It suffices to show that if $g_{\alpha}(x, M) \neq 0$ on $\alpha_{M}$ and $\alpha_{M} \cap \beta_{M} \neq \varnothing$ then $g_{\beta}(x, M) \neq 0$ on $\beta_{M}$. This follows because $f_{\alpha}, g_{\alpha}$ (resp. $f_{\beta}, g_{\beta}$ ) are relatively prime on $\alpha$ (resp. $\beta$ ), and hence $f_{\beta}=u_{\alpha \beta} f_{\alpha}$ and $g_{\beta}=u_{\alpha \beta} g_{\alpha}$ on $\alpha \cap \beta$ and thus on $\alpha_{M} \cap \beta_{M}$, where $u_{\alpha \beta}: \alpha \cap \beta \rightarrow \mathbb{C}$ is non-vanishing. Since $\hat{U}$ is connected there exists a finite chain of open balls $\beta_{M} \neq \varnothing$ "connecting" $\alpha_{M}$ with $U$. Hence, $g_{\alpha} \neq 0$ on each $\alpha_{M} \neq \varnothing$.

$$
\text { We extend } \hat{F}_{M} \text { to } \tilde{U} \text { by setting } \tilde{F}_{M}:=\operatorname{pr} * \hat{F}_{M} \text {. }
$$

Lemma A3. $\tilde{F}_{M}$ and $\tilde{K}_{M}$ are in involution on $\tilde{U}$.

Proof. Let $p \in \tilde{U}$ and let $W \subset \tilde{U}$ be a neighborhood of $p$ such that the restriction of pr to $W$ is a diffeomorphism. Then there exist $\hat{K}_{M}^{W}: \operatorname{pr}(W) \rightarrow \mathbb{C}$ such that $\operatorname{pr}^{*} \hat{K}_{M}^{W}=$ $\left.\widetilde{K}_{M}\right|_{W}$. Since pr is symplectic we have $\left\{\widetilde{F}_{M}, \widetilde{K}_{M}\right\}(p)=\left\{\hat{F}_{M}, \hat{K}_{M}^{W}\right\}(\operatorname{pr}(p))$ as long as $p$ does not project onto the singular set associated with $\hat{F}_{M}$. Notice that the last bracket expression vanishes at points $p \in \tilde{U}$ which project onto real points of $\hat{U}$ (i.e., onto points of $U$ ) and for which $w=\sqrt{\rho^{2}+X_{3}^{2}}$ (positive square root). The statement of the lemma follows by invoking the identity theorem for analytic functions. Here it is important to realize that the singular set of $\hat{F}$ has complex codimension one in $\hat{U}$ so that removing it from $\hat{U}$ still leaves a connected set.

\section{Appendix B: Sufficient Conditions for the Monodromy Group of a Second Order Linear Fuchsian Equation not to be Ziglin}

In [22] Kovacic's algorithm [27] is used to derive necessary and sufficient conditions for the monodromy group of a second order linear Fuchsian equation not to be Ziglin (see also [31]). Here, we only present that part of Kovacic's algorithm 
which is employed in the main body of the paper to conclude that the monodromy group of our NVE is not Ziglin.

Consider the second-order differential equation

$$
\xi^{\prime \prime}+p(\rho) \xi^{\prime}+q(\rho) \xi=0,
$$

viewed on the Riemann sphere $\mathbb{P}^{1}$. Since (B1) is Fuchsian, $p$ and $q$ have a representation

$$
\begin{aligned}
& p(\rho):=\sum_{k=0}^{\ell} \frac{A_{k}}{\rho-a_{k}}, \\
& q(\rho):=\sum_{k=0}^{\ell} \frac{B_{k}}{\left(\rho-a_{k}\right)^{2}}+\sum_{k=0}^{\ell} \frac{C_{k}}{\rho-a_{k}}, \quad \sum_{k=0}^{\ell} C_{k}=0,
\end{aligned}
$$

where $\ell$ is some nonnegative integer and the $A_{k}$ 's, $B_{k}$ 's, $C_{k}$ 's are complex constants. By means of the transformation $\xi=\zeta \exp \left(-\frac{1}{2} \int p d \rho\right)$ Eq. (B1) can be transformed to normal form:

$$
\zeta^{\prime \prime}=r(\rho) \zeta
$$

where

$$
\begin{aligned}
r(\rho) & :=-q(\rho)+\frac{1}{4} p(\rho)^{2}+\frac{1}{2} p^{\prime}(\rho) \\
& =\sum_{k=0}^{\ell} \frac{\beta_{k}}{\left(\rho-a_{k}\right)^{2}}+\sum_{k=0}^{\ell} \frac{\delta_{k}}{\rho-a_{k}}, \quad \sum_{k=0}^{\ell} \delta_{k}=0 .
\end{aligned}
$$

Here,

$$
\beta_{k}=\frac{1}{4}\left[\left(1-A_{k}\right)^{2}-4 B_{k}-1\right], \quad \delta_{k}=-C_{k}+\frac{1}{2} A_{k} \sum_{j \neq k=0}^{\ell} \frac{A_{j}}{a_{k}-a_{j}} .
$$

We also define

$$
\beta_{\infty}=\frac{1}{4}\left[\left(1-A_{\infty}\right)^{2}-4 B_{\infty}-1\right]
$$

where

$$
A_{\infty}=\sum_{k=0}^{\ell} A_{k}, \quad B_{\infty}=\sum_{k=0}^{\ell}\left(B_{k}+C_{k} a_{k}\right) .
$$

The following theorem can be directly read off from Theorems 3.5, 4.9, 4.13, and 4.18 of [22].

Theorem B. Suppose that the constants $\beta_{k}, \beta_{\infty}, \delta_{k}, A_{\infty} B_{\infty}$ in (B5), (B6) are such that by going through the algorithm described below none of the mutually exclusive cases. I, II, III can be realized successfully. Then the monodromy groups of (B3) and (B1) are not Ziglin.

Idea of the Proof. Kovacic's algorithm tests a second order linear ODE (in normal form) systematically for the nature of its solutions. In the first three parts it searches 
for a solution of type $\exp \int \theta$, with $\theta$ algebraic over the field $\mathbb{C}(\rho)$. They differ by the nature of $\theta$ as follows:

(I) $\theta \in \mathbb{C}(\rho)$,

(II) $\theta$ algebraic of degree 2 over $\mathbb{C}(\rho)$,

(III) $\theta$ algebraic of degree $4,6,12$ over $\mathbb{C}(\rho)$.

In the fourth case which holds when the algorithm decides that the first three cases fail no "closed form solution" exists. The four cases also differ by the nature of the differential Galois group $G_{D}$, associated with the differential equation (which in all cases is an algebraic subgroup of $\operatorname{SL}(2, \mathbb{C})$ ) namely as follows:

(I) $G_{D}$ is triangulizable.
(II) $G_{D}$ is conjugate to a subgroup of $D \cup D J, J=\left[\begin{array}{cc}0 & 1 \\ -1 & 0\end{array}\right]$ with at least one element in $D J$ so that case I does not hold. Here $D=$ diagonal subgroup of $\mathrm{SL}(2, \mathbb{C})$.

(III) $G_{D}$ is a finite group and cases (I) and (II) do not hold, i.e., $G_{D} /(1,-1)$ is either tetrahedral (order 24), octahedral (order 48) or icosahedral (order 120).

(IV) $G_{D}=S L(2, \mathbb{C})$.

For our purpose the goal is to show that the algorithm decides against the realization of the first three cases since in this case the differential Galois group $G_{D}$ of the Fuchsian ODE (B3) is $\operatorname{SL}(2, \mathbb{C})$. In turn this implies that the monodromy group of (B3) is not Ziglin since otherwise its Zariski closure, $G_{D}$ would have this property. (See Proposition 3.1 (c), Proposition 2.2(d) of [22]). It follows from Theorem 3.5 (3) of [22] that also the monodromy group of (B1) is not Ziglin (see also Prop. 4.25 of [31]). The tests of the algorithm for the first three cases are as follows:

Case I

(1) For $k=1,2, \ldots, \ell$ define the numbers

$$
\begin{gathered}
\alpha_{k}^{ \pm}= \begin{cases}\frac{1}{2}\left\{1 \pm\left[1+4 \beta_{k}\right]^{1 / 2}\right\} & \text { if } \beta_{k} \neq 0 \\
1 & \text { if } \beta_{k}=0 \text { and } \delta_{k} \neq 0 \\
0 & \text { if } \beta_{k}=\delta_{k}=0\end{cases} \\
\alpha_{\infty}^{ \pm}=\frac{1}{2}\left\{1 \pm\left[1+4 \beta_{\infty}\right]^{1 / 2}\right\} .
\end{gathered}
$$

(2) Choose plus or minus signs $s(k), s(\infty)$ such that

$$
d_{I}:=\alpha_{\infty}^{s(\infty)}-\sum_{k=0}^{\ell} \alpha_{k}^{s(k)}
$$

is a nonnegative integer, and

(3) To each such choice search for a unique monic polynomial $P_{I}$ (i.e., having leading term equal to unity) of degree $d_{I}$ satisfying the ODE

$$
P_{I}^{\prime \prime}+2 \omega P_{I}^{\prime}+\left(\omega^{\prime}+\omega^{2}-r\right) P_{I}=0,
$$


where

$$
\omega=\omega(\rho)=\sum_{k=0}^{\ell} \frac{\alpha_{k}^{s(k)}}{\rho-a_{k}} .
$$

Case II.

(1) For each $k=0, \ldots, \ell$ define the sets

$$
\left.\begin{array}{rl}
E_{k} & =\left\{2+e\left(1+4 \beta_{k}\right)^{1 / 2}: e=0, \pm 2\right\} \cap \mathbb{Z} \text { if } \beta_{k} \neq 0, \\
E_{k} & =\{4\} \text { if } \beta_{k}=0 \text { and } \delta_{k} \neq 0, E_{k}=\{0\} \text { if } \beta_{k}=0 \text { and } \delta_{k}=0,
\end{array}\right\}
$$

(2) Choose numbers $e_{k} \in E_{k}(k=0, \ldots, \ell)$ and $e_{\infty} \in E_{\infty}$ which are not all even integers and are such that

$$
d_{I I}:=\frac{1}{2}\left[e_{\infty}-\sum_{k=0}^{\ell} e_{k}\right]
$$

is a nonnegative integer.

(3) To each such choice search for a monic polynomial $P_{I I}$ of degree $d_{I I}$ satisfying the ODE

$$
P_{I I}^{\prime \prime \prime}+3 \theta P_{I I}^{\prime \prime}+\left(3 \theta^{\prime}+3 \theta^{2}-4 r\right) P_{I I}^{\prime}+\left(\theta^{\prime \prime}+3 \theta \theta^{\prime}+\theta^{3}-4 r \theta-2 r^{\prime}\right) P_{I I}=0
$$

where

$$
\theta(\rho)=\frac{1}{2} \sum_{k=0}^{\ell} \frac{e_{k}}{\rho-a_{k}}
$$

Case III.

(1) For $n=4,6,12$ and $k=0, \ldots, \ell$ define the sets

$$
\left.\begin{array}{l}
F_{k}(n)=\left\{6+(12 e / n)\left(1+4 \beta_{k}\right)^{1 / 2}: e=0, \pm 1, \ldots, \pm(n / 2)\right\} \cap \mathbb{Z} \\
\text { if } \beta_{k} \neq 0, \quad F_{k}(n)=\{12\} \quad \text { if } \beta_{k}=0 \text { and } \delta_{k} \neq 0, \\
F_{k}(n)=\{0\} \text { if } \beta_{k}=\delta_{k}=0,
\end{array}\right\}
$$

and

$$
F_{\infty}(n)=\left\{6+(12 e / n)\left(1+4 \beta_{\infty}\right)^{1 / 2}: e=0, \pm 1, \ldots, \pm(n / 2)\right\} \cap \mathbb{Z} .
$$

(2) Going through the cases $n=4,6,12$ choose numbers $f_{k}(n) \in F_{k}(n)$ $(k=0, \ldots, \ell)$ and $f_{\infty}(n) \in F_{\infty}(n)$ such that

$$
d_{I I I}(n):=\frac{n}{12}\left[f_{\infty}(n)-\sum_{k=0}^{\ell} f_{k}(n)\right]
$$

is a nonnegative integer. 
(3) For each such choice define the function

$$
\theta_{n}(\rho)=\frac{n}{12} \sum_{k=0}^{\ell} \frac{f_{k}(n)}{\rho-a_{k}}
$$

and search for a monic polynomial $P_{I I I}$ of degree $d_{I I I}(n)$ with the following property: If polynomials $P_{i}(i=n, n-1, \ldots, 0,-1)$ are defined recursively starting from $P_{I I I}$ using the formulae

$$
\begin{aligned}
P_{n} & :=-P_{I I I}, \\
P_{i-1} & :=-S P_{i}^{\prime}+\left[(n-i) S^{\prime}-S \theta_{n}\right] P_{i}-(n-i)(i+1)+S^{2} r P_{i+1},
\end{aligned}
$$

then necessarily $P_{-1} \equiv 0$. Here, $r$ was defined in (B3) and $S$ is the expression

$$
S(\rho)=\prod_{k=0}^{\ell}\left(\rho-a_{k}\right) .
$$

\section{References}

1. Robnik, M.: J. Phys. A., Math. Gen. 14, 3195 (1981)

2. Robnik, M.: J. de Phys., Coll. C2, suppl. to No. 11, 43, 45 (1982)

3. Reinhardt, W.P., Farrely, D.: J. de Phys., Coll. C2, suppl. to No. 11, 43, 29 (1982)

4. Atomic Spectra and Collisions in External Fields, Taylor, K.T., Nayfeh, M.H., Clark, E.W. (eds.). New York, London: Plenum, 1989

5. Atoms in Strong Fields, Nicolaides, C.A., Clark, C.W., Nayfeh, M.H. (eds.), NATO ASI Series 212. New York, London: Plenum, 1990

6. Percival, I.C.: J. Phys. A., Atom Molec. Phys. 6, L229 (1973)

7. Percival, I.C.: Lecture Notes in Physics 93. Berlin, Heidelberg, New York: Springer, 1979, p. 259

8. Berry, M.V.: J. Phys. A., Math. Gen. 10, 2083 (1977)

9. Berry, M.V.: Chaotic Behavior of Deterministic Systems (Les Houches, Session 36), Ioss, G., Helleman, R.G.H., Stora, P. (eds.) Amsterdam: North Holland, 1983, p. 171

10. Berry, M.V.: Proc. Roy. Lond. A 400, 229 (1985)

11. Ozorio de Almeida, A.M.: Hamiltonian Systems: Chaos and Quantization. Cambridge: Cambridge University Press, 1988

12. Delande, D.: Chaos and Quantum Physics (Les Houches, Session 52), Giannoni, M.-J., Voros, A., Zinn-Jiustin, J. (eds.). Amsterdam: North Holland, 1991, p. 665

13. Streit, L., Vázquez, L., Vilela Mendes, R.: Essays in Classical and Quantum Dynamics (A.W. Sáenz Festschrift volume), Ellison, J.A., Überall, H. (eds.). Philadelphia: Gordon Breach Science Publishers, 1991, p. 129

14. Dragt, A.J., Finn, J.M.: J. Geophys. Res. 81, 2327 (1976)

15. Dragt, A.J., Finn, J.M.: J. Math. Phys. 20, 2649 (1979)

16. Finn, J.M.: Integrals of canonical transformations and normal forms for mirror machine Hamiltonians, Ph.D. thesis, University of Maryland, College Park, MD (1974)

17. Ziglin, S.L.: Funct. Anal. Appl. 16, 1818 (1982); 17, 6 (1983)

18. Ito, H.: J. Appl. Math. Phys. (ZAMP) 38, 459 (1987)

19. Yoshida, H.: Physica 21 D, 163 (1986)

20. Yoshida, H.: Physica 29 D, 128 (1987)

21. Yoshida, H.: Proc. of the Workshop on Finite-Dimensional Integrable Nonlinear Dynamical Systems, Leach, P.G.L., Steeb, W.H. (eds.). Singapore: World Scientific, 1988, p. 74

22. Churchill, R.C., Rod, D.L.: Siam J. Math. Anal. 22, 1970 (1991)

23. Baider, A., Churchill, R.C., Rod, D.L.: J. Dynamics Differential Equations 2, 451 (1990)

24. Rod, D.L.: Hamiltonian Dynamical Systems. Meyer, K.R., Saari, D.C. (eds.), Contemporary Mathematics 81. Providence, R.I.: American Mathematical Society, 1988, p. 259 
25. Rod, D.L., Churchill, R.C.: Proc. of the Workshop on Finite-Dimensional Integrable Nonlinear Dynamical Systems, Leach, P.G.L., Steeb, W.H. (eds.). Singapore: World Scientific, 1988, p. 94

26. Churchill, R.C., Rod, D.L.: J. Differential Equations 76, 91 (1988)

27. Kovacic, J.: J. Symbolic Computation 2, 3 (1986)

28. Kaplanski, I.: An Introduction to Differential Algebra (2nd ed.). Paris: Hermann, 1976

29. Singer, M.F.: Computer Algebra and Differential Equations. Tournier, E. (ed.). New York: Academic Press, 1988, p. 3

30. Tretkoff, C., Tretkoff, M.: Am. J. Math. 1010, 1327 (1979)

31. Baider, A., Churchill, R.C., Rod, D.L., Singer, M.F.: On the infinitesimal Geometry of integrable systems, to be published by the Fields Institute, Ontario

Communicated by J.-P. Eckmann 
